\title{
VISIONAIR VISION Advanced Infrastructure for Research
}

\author{
Andreas Kopecki, Uwe Wössner \\ HLRS, University of Stuttgart \\ \{kopecki,woessner\}@hlrs.de
}

\author{
Lionel Roucoules \\ Arts et Métiers ParisTech Institute \\ Lionel.Roucoules@ensam.eu \\ Daniel Bündgens \\ RWTH Aachen \\ buendgens@vr.rwth-aachen.de
}

Frédéric Noël, Cedric Masclet
INP Grenoble

frederic.noel@g-scop.fr, cedric.masclet@grenoble-inp.fr

\author{
Dimitris Mavrikios, Loukas Rentzos \\ LMS, University of Patras \\ \{mavrik,rentzos\}@1ms.mech.upatras.gr
}

Okung-Dike Ntofon, Martin Reed

NLM, University of Essex

ontofo,mjreed@essex.ac.uk

\author{
Andrzej Milecki \\ Poznan University of Technology \\ andrzej.milecki@put.poznan.pl
}

\author{
Christian Weidig \\ University of Kaiserslautern \\ weidig@ cpk.uni-kl.de \\ Georges Dumont \\ ENS Cachan/IRISA-INRIA \\ Georges.Dumont@irisa.fr \\ Peter Baranyi \\ MTA SZTAKI \\ baranyi@sztaki.hu
}

Marco Attene, Franca Giannini, Michela Spagnuolo
CNR-IMATI
$\{$ attene,giannini,spagnuolo $@ @$ ge.imati.cnr.it

\begin{abstract}
The development of information technologies, the increasing complexity of the information to be handled and analysed along with the increasing capacities in scientific and engineering simulations call for the development of increasingly powerful visualisation tools and methods. By integrating existing facilities, VISIONAIR is aimed at creating a world-class research infrastructure that will be open to research communities across Europe and around the world for conducting state-of-the-art research.
\end{abstract}

\section{INTRODUCTION}

VISIONAIR [1] is establishing a European infrastructure for high level visualisation facilities that is open to research communities across Europe and around the world. By integrating existing facilities to a pan-European network, it will create a world-class research infrastructure enabling to conduct cuttingedge research. Current scientific challenges such as climate evolution, environmental risks, molecular biology, health, and energy require the management of increasingly complex and voluminous information, thus calling for the development of ever more powerful visualisation methods and tools. On many sites across Europe, it is infeasible to fund the necessary visualisation facilities that are needed to tackle high fidelity, large screen and/or immersive visualisation. VISIONAIR is targeted to fill in this gap by providing access to the partner facilities, opening its doors for interested researchers to use the multitude of services available across the European visualisation facilities. After submitting a successful research proposal, international researchers are invited to visit the partner facility of their choice or what fits best to the scientific goals they have in mind to conduct their research. They are not only given access to the top visualisation facilities in Europe, but are also supported in their research by funding their living and travel expenses. Researchers can choose from over 20 facilities located in 12 countries in Europe and Israel.

The project targets different fields of visualisation. Scientific Visualisation offers access to methods, software and hardware needed for successfully visualising scientific data, including - but not limited - to engineering, medical visualisation, biology, chemistry and physics. Ultra-High-Definition facilities connected by high speed networks are targeted at users that want to create high resolution, high quality images (up to $8 \mathrm{k}$ ) and possibly access those by high speed networks. VISIONAIR provides the hardware and the unique network distribution services needed for transmission of the images to their end-points. The network services enable multiple high-resolution digital-media streams to be transported among global sites, using dynamically provisioned optical light paths across multiple domains, which can be used on a scheduled or on-demand basis. While Scientific and Ultra-High-Definition Visualisation can be done in any environment, researchers specifically targeting Virtual Reality can also apply at a multitude of facilities. Here, the focus is on immersive - possibly also haptic - experiences in virtual environments. Equipment available for researchers ranges from head mounted displays to fully fledged stereoscopic PowerWalls and CAVEs. Further specialised equipment available allows to carry out research by using Augmented Reality, a technique that enables users to overlay the real environment with context dependent computer generated images. Researchers also have access to the latest developments in display technology, like holographic displays or the above mentioned 8k displays.

The project maintains an already huge database of visualisation software and models that is available for all researchers for free. Thus, experts can explore the multitude of visualisation 


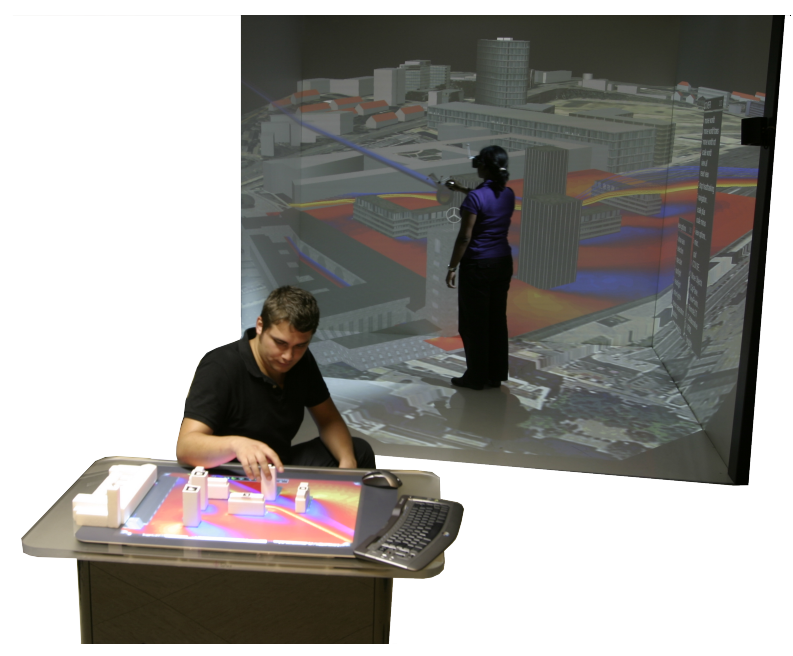

Fig. 1. CAVE and MultiTouch Table at the HLRS

packages that are already available. Software covered here ranges from processing filters, converters and readers to fully fledged modellers and visualisation packages. VISIONAIR is rounded up by several research activities concerning the usability and accessibility of the facilities and their software for external researchers with a strong focus on collaboration.

By creating this European visualisation network it will be possible to create a landmark that will have a broad visibility throughout the research communities around the world. In the following pages we introduce some of the facilities participating in the network to give researchers an impression about the possibilities opening up with VISIONAIR, while inviting all interested parties to submit proposals to use the VISIONAIR platform for their research. Not all partners found space in this paper, so you are invited to visit the VISIONAIR homepage for an overview over all partners.

\section{TRAnS National ACCESS}

\section{A. HLRS, University of Stuttgart}

The HLRS is the first national high performance computing centre in Germany. Within this framework, the HLRS provides access to its CAVE [2], PowerWalls, head mounted displays (HMD) and its haptic driving simulator. We also offer several input devices ranging from optical tracking to tangible interfaces and MultiTouch tables (Fig. 1). Visitors will be able to interactively visualise large simulation results or even realise computational steering and interactive simulations by leveraging the power of a 40 nodes visualisation cluster. The visualisation software COVISE [3] will not only allow visitors to analyse their data in Virtual Reality but they can also overlay physical prototypes or test beds with their visualisation using Augmented Reality techniques [4]. The collaborative features will allow them to analyse the simulations together with their colleagues at home or with remote scientists.

\section{B. LMS, University of Patras}

The Laboratory for Manufacturing Systems and Automation (LMS) [5] is part of the Department of Mechanical Engi- neering and Aeronautics, at University of Patras, in Greece. LMS is oriented to research and development in cutting edge scientific and technological fields. LMS hosts three research groups involved in innovative manufacturing processes, advanced human-centred design techniques and production systems planning and networking. Within VISIONAIR, LMS provides two visualisation infrastructures. The firstis based on immersive visualisation and interaction technologies that promote the human-centred simulation in the areas of manufacturing and engineering. The immersive system consists of HMD devices, stereoscopic glasses, magnetic motion tracking systems and interaction devices that are capable of providing a high quality immersive experience. The second infrastructure provides a state-of-the-art holographic display that projects airborne images for high fidelity visualisation and interactive presentation of virtual prototypes, aiming at mutli-user environments. The system is based on the EON Reality ICrystal device that offers high Definition (1280x768) holograms through a 50" wide holographic display. The system is able to provide intuitive interaction through wireless devices such as a wand and keyboard. Collaborative design software packages are also provided to visitors, in order to experience navigation and interaction within shared virtual environments for multiusers.

\section{TU Kaiserslautern}

The University of Kaiserslautern is using a CAVE for research and visualisation of machining processes and production planning in the field of Mechanical Engineering. This CAVE, the modelling and simulation software driving it and our expertise will be provided to our visitors. But our offer is not limited to an engineering point of view. With our high immersive system, any other Scientific Visualisation and Virtual Reality topic can be addressed, also. The potential of interaction and manipulation within virtual scenes in 1:1 and the visualisation of huge data-sets complete the opportunity we offer.

\section{Arts et Métiers ParisTech Institute}

Métiers ParisTech Institute (AMPT) is a prestigious higher education engineering school. It has trained over 85,000 engineers since its founding in 1780 by the Duke de La Rochefoucauld Liancourt. The research activities of Arts et Métiers Institute is broken down into three topics that are: Mechanics, materials and production, fluids and energetic systems, and Design, industrialisation, risks and decisions. Arts et Métiers ParisTech contributes their virtual reality facilities to the VISIONAIR infrastructure. We offer a 4 walls CAVE system, a human scale haptic feedback system (Spidar), 3D sound rendering technologies, and a 6 DOF dynamic platform (driving simulation platform).

\section{E. NLM, University of Essex}

VR applications are set to benefit from the emergence of Ultra-High-Definition (UHD) digital media formats as these formats provide viewers with a more convincing impression 


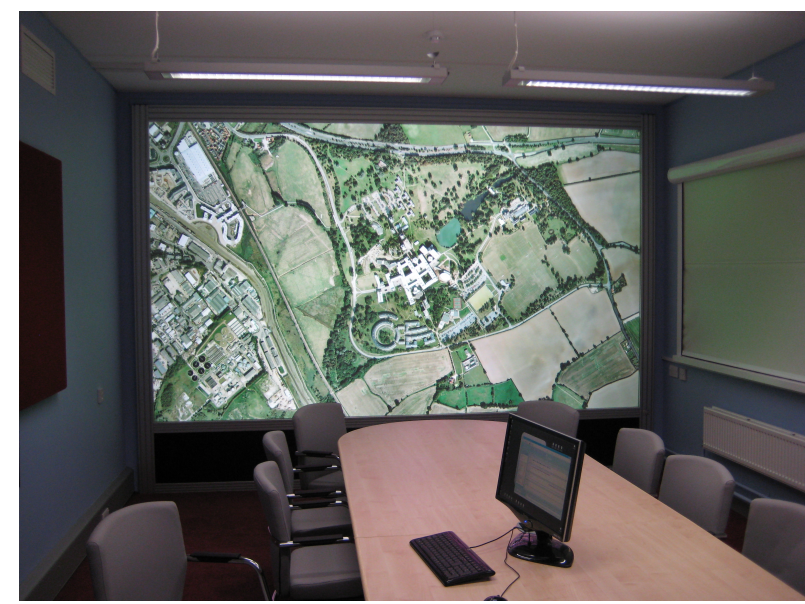

Fig. 2. 8k Auditorium at University of Essex

of reality and greater sense of presence compared to other existing digital media formats. The NML [6] is an interdisciplinary laboratory focused on research and development for the deployment of UHD media applications over high performance communication networks. It is one of the few network connected UHD facilities in the world and is equipped with visualisation facilities for emerging $4 \mathrm{~K} 3 \mathrm{D}$ and $8 \mathrm{~K}$ media formats. Within the VISIONAIR framework, the NML is providing advanced network technologies for application-aware delivery of future networked UHD applications (including UHD VR applications) and remote collaborations between researches. In addition, it is providing access to its visualisation facilities as well as its extensive network infrastructure comprising connections to UK, European and International locations through 1 and 10 Gbps lightpath connectivity.

\section{F. INRIA (Institut National de Recherche en Informatique et en Automatique)}

INRIA, the French national institute for research in computer science and control, operating under the dual authority of the Ministry of Research and the Ministry of Industry, is dedicated to fundamental and applied research in information and communication science and technology (ICST). The Institute also plays a major role in technology transfer by fostering training through research, diffusion of scientific and technical information, development, as well as providing expert advice and participating in international programs. INRIA is represented by two infrastructures within the VISIONAIR network and thus associated to other academic partners.

The VR4i, Virtual Reality for Improved Innovative Immersive Interaction, team is located at Rennes together with IRISA. The main purpose of the VR4i team is to improve the interaction of users with and through virtual worlds. Our main research activity is concerned with real-time simulation of complex dynamic systems, and we investigate real-time interaction between users and these systems. We address mechanical simulation, control of dynamic systems, realtime simulation, haptic interaction, multimodal interaction,

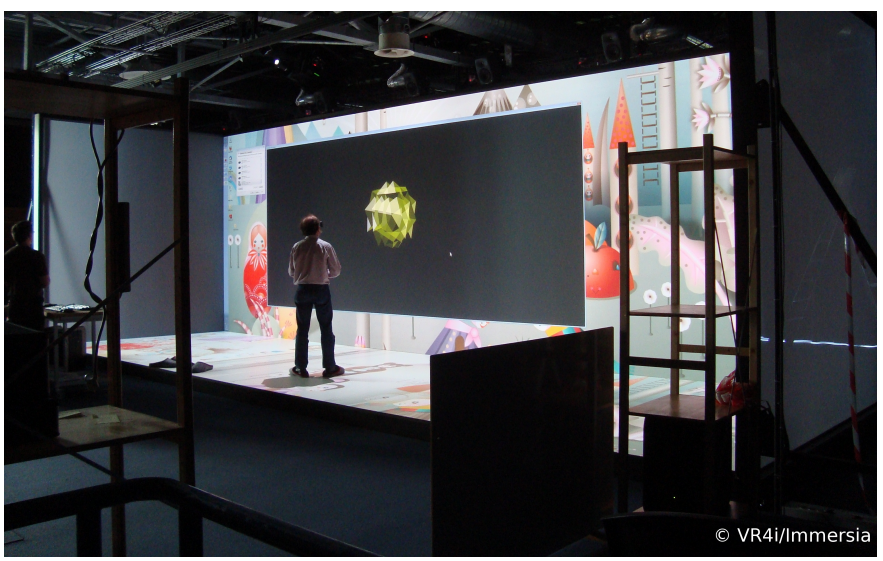

Fig. 3. One of the CAVEs at INRIA

collaborative interaction and modeling of virtual environments. We want to allow real users to interact in Virtual Reality as naturally as in reality.

The MOAIS team is located in Grenoble and is a joint research team shared by INRIA, UJF (Universite Joseph Fourrier) and Grenoble-INP (Institut Polytechnique de Grenoble). MOAIS focuses on the programming of applications where increasing the number of resources is a key to improve performance: beyond the optimisation of the application itself, the effective use of a larger number of resources is expected to enhance the performance. This encompasses large scale scientific interactive simulations (e.g. immersive virtual reality) that involve various resources: input (sensors, cameras, ...), computing units (processors, memory), output (videoprojectors, images wall) that play a prominent role in the development of high performance parallel computing.

\section{G. RWTH Aachen}

The Virtual Reality Group at RWTH Aachen University provides access to its distinctive infrastructure which includes several desktop-sized VR systems, a PowerWall display, a dual-screen L-bench setup, and a 5-sided CAVE system. In order to use these systems, visitors will have access to the VR toolkit ViSTA and its add-on libraries, e.g. ViSTA Flowlib. In addition, they will be offered the help of the Virtual Reality Group's visualisation experts who have established a long standing track record of successful, interdisciplinary cooperations [7]. Consequently, visitors will be able to produce interactive, immersive visualisations based on complex, large datasets, where a particular focus lies on the interactive exploration of time-varying data [8].

\section{H. Poznan University of Technology}

The Virtual Design and Automation (VIDA) Laboratory [10] operates in the Faculty of Mechanical Engineering and Management at Poznan University of Technology (PUT). It is equipped with advanced Virtual Reality systems - two large screen stereo displays (with passive and active stereoscopy), high-class HMD and a number of interaction devices, like wireless optical and magnetic tracking systems 
and gesture recognition gloves (allowing intuitive interaction with 3D applications). The laboratory is also equipped with high end haptic devices that provide feedback on the forces occurring during operator interaction with virtual objects. The advanced haptic devices with force feedback allow the precise manipulation in VR environments. The research performed in the laboratory covers mainly the application of aforementioned VR systems in production engineering, development of control systems of production devices and systems, and also in ergonomics and medicine.

\section{MTA SZTAKI-3DICC Lab}

The 3DICC Lab (3D Internet based Control and Communication Lab) has the first and largest 3D immersive virtual system in Hungary with various motion capture systems, robots and a 4D video studio. The laboratory also has a VirCA (Virtual Collaboration Arena [11]) platform that is capable of connecting and integrating real/virtual and software/hardware components from different laboratories in universities or research institutes through the use of networks. The user can interact with the whole integrated system via a $3 \mathrm{D}$ virtual word or even collaborate with other users from different laboratories. Visitors will be able to get practice on the system and connect their laboratories, robots and software components to the platform for visualisation or human interaction and collaboration purposes. In the same time the visitors who are on the platform are free to remotely integrate all existing components developed at different laboratories into the system, and also have the chance to integrate their methodologies into various robots of other laboratories.

\section{J. Institut Polytechnique de Grenoble}

The G-SCOP laboratory belonging to Grenoble Institute of Technology is a multi-disciplinary laboratory aiming to face the new scientific challenges associated with production systems. Product design, production system management and mathematics optimisation are the core competencies of the laboratory. In the field of product design, product representation and visualisation, collaborative interactions and activity based design theories are major issues of this laboratory. Within the global GINOVA platform the laboratory develops the MEXICO platform which is a registering studio for analysing usage of visualisation and interaction devices. The impact of new devices (holographic screen from holografika, multi-touch table (DiamondTouch) with collaboration identification capacity, a large stereoscopic screen and haptic devices (Phantom arm) and tracking systems) on usage in innovative scientific applications may be undertaken. This platform, connected to a visio-conference system managed by G-SCOP, will be open to access within the trans-national access activities for all visiting scientists.

\section{ViRTUAL ACCESS}

Typically, preparing digital geometry for visualisation within VR facilities requires diverse software tools and, if an authoring phase is included, using existing geometry instead of starting from scratch can be highly desirable. Visualization Virtual Services (VVSs) at CMR-IMATI provide access to virtual facilities through a remote connection. Based on the Digital Shape Workbench [9] paradigm developed within the AIM@SHAPE project, the virtual facilities are provided as online services that allow to share digital shapes and software tools. The VVSs act as an e-Science framework in terms of an operational, distributed and web-based software system. By providing search services for both 3D shapes and software tools, the VVSs offer a valid support for applications that have to deal with the processing and visualisation of digital shapes, with a particular focus to 3D. While providing facilities for the retrieval of useful processing software and geometric shapes, the VVSs support the creation of original digital shapes (2D images, videos, 3D shapes, and higher-dimensional geometric models) that will be possibly analysed, enriched and combined with other data, with the objective of providing useful information to be visualised.

\section{Project Calls}

Project calls are expected to open end of 2011. Interested researchers are invited to submit a proposal at http://www.infra-visionair.eu/. The partners participating in the VISIONAIR network are:

INPG Grenoble $[\mathrm{F}] \bullet$ Grenoble INP $[\mathrm{F}] \bullet$ University of Patras [GR] • Cranfield University [UK[ $\bullet$ Universiteit Twente [NL] - Universität Stuttgart [D] • PSNC Posnan [PL] • Université de la Mediterrané Marseille [F] • CNR Genova [I] • INRIA Rennes [F] • KTH Stockholm [S] • Technion Haifa [IL] • RWTH Aachen [D] • Poznan University of Technology [PL] - Arts et Métiers ParisTech Institute [F] • TU Kaiserslautern [D] • University of Salford [UK] • Fraunhofer IPK Berlin [D] • i2cat Barcelona [ES] • University of Essex Colchester $[\mathrm{UK}] \bullet$ MTA SZTAKI Budapest [HU] • ECN Nantes [F] • University College London [UK] • Politecnico di Milano [I] - EMIRACLE Brussels [B]

\section{ACKNOWLEDGMENT}

VISIONAIR is partially funded by the EC under the 7th Framework Programme.

\section{REFERENCES}

[1] The VISIONAIR web page http://www.infra-visionair.eu/

[2] http://www.hlrs.de/organization/av/vis/research/vr/velab/

[3] http://www.hlrs.de/covise

[4] http://www.hlrs.de/organization/av/vis/research/ar/

[5] http://lms.mech.upatras.gr/

[6] http://hpn.essex.ac.uk/facilities/netmediafacilities.php?mn=fa

[7] B. Hentschel, I. Tedjo, M. Probst, M. Wolter, M. Behr, C. Bischof and T. Kuhlen: Interactive blood damage analysis for ventricular assist devices. IEEE Transactions on Visualization and Computer Graphics 14(6):15151522, 2008.

[8] M. Wolter, I. Assenmacher, B. Hentschel, M. Schirski and T. Kuhlen: A time model for time-varying visualization. Computer Graphics Forum 28(6), pp. 1561-1571, 2009

[9] http://dsw.aimatshape.net/

[10] http://www.vida.put.poznan.pl/en/lab_vr.php

[11] Virtual Collaboration Arena http://www.virca.hu/ 